\title{
Ultrasmall Tungsten Carbide Catalysts Stabilized in Graphitic Layers for High-performance Oxygen Reduction Reaction
}

Junjie Guo, ${ }^{a * \#}$ Zhe Mao, ${ }^{b \#}$ Xiaoli Yan, ${ }^{a}$ Rui Su, ${ }^{c}$ Pengfei Guan, ${ }^{c^{*}}$ Bingshe Xu, ${ }^{a}$ Xuefeng Zhang, ${ }^{b^{*}}$ Gaowu Qin $^{b}$ and Stephen J. Pennycook ${ }^{d}$

${ }^{a}$ Key Laboratory of Interface Science and Engineering in Advanced Materials of Ministry of Education, Taiyuan University of Technology, Taiyuan 030024, P. R. China

${ }^{b}$ Key Laboratory for Anisotropy and Texture of Materials (MOE), School of Materials Science and Engineering, Northeastern University, Shenyang 110819, P. R. China

${ }^{c}$ Beijing Computational Science Research Center, Beijing 100193, P. R. China

${ }^{\mathrm{d}}$ Department of Materials Science and Engineering, National University of Singapore, Singapore 117576, Singapore

KEYWORDS: tungsten carbide nanoparticles; graphitic layers; electron microscopy; electrocatalysts; oxygen reduction reaction

*Corresponding Author; ${ }^{\#}$ Equal contribution to this work.

E-mail: guojunjie@tyut.edu.cn(J.Guo)

pguan@csrc.ac.cn (P.Guan)

zhangxf@atm.neu.edu.cn (X. Zhang) 


\begin{abstract}
Tungsten carbide (WC) has been proven Pt-like features in many catalytic reactions, and presents promising potentials to substitute noble metal-based electrocatalysts. However, the catalytic performances cannot be improved to the industrial standard due to the low density of catalytically active sites that are relevant to the size and stability of catalysts. Herein, we synthesized WC catalystswith a mean diameter of $1.9 \pm 0.9 \mathrm{~nm}$, comprising a high density of single $\mathrm{W}$ atoms, sub-nanometer and nanometer WC clusters, which are completely encapsulated in high-defective graphitic layers (WC@C). Such highdefective graphitic layersnot only improve the overall conductivity for accelerating the penetration and exchange of ions and electrons during the electrocatalytic process of oxygen reduction, but also suppress the chemical/thermal coarsening of WC catalysts, thus providing excellent electrocatalytic performances in four-electron transfer process for oxygen reduction reaction.
\end{abstract}

\title{
1. Introduction
}

The oxygen reduction reaction (ORR) is the most important reaction in energy converting systems such as polymer electrolyte fuel cells (PEFC).[1]However, the energy converting efficiency and power density are seriously limited by oxygen reduction rate that is closely related to cathode catalysts utilized, for example, on a Pt electrode the exchange current density of hydrogen oxidation is several orders higher than that of ORR.[2]Therefore, development of highperformance cathode catalysts has been attracting considerable attention.[3-5]Currently, noble metal-based catalysts have exhibited the best overall performance;[6-10]nevertheless, with the cost, scarcity and tolerance, researchers have realized the importance on the exploration of 
substitutes. Among all the candidates, carbides of groups IV through VI have been proven Pt-like catalytic features.[11-13]For examples, they have been applied in catalyzing decomposition of methanol,[14]dehydrogenation of butane,[15]as well as hydrogenation and dechlorination of Freon[16]. However, in the electrochemical catalysis reactions, carbides themselves cannot present desirable activity for oxygen reduction in alkaline atmospheres;[17] therefore they were only used as promoters for the synergistic catalytic effect together with noble-based catalysts.[18, 19]

Although it has been well recognized that the smaller catalyst exhibits the higher catalytic activity, but the coarsening of catalysts during the reaction process, which results in undesirable chemical and structural stability, cannot be effectively resolved yet.[20-25]On the other hand, the traditional techniques for synthesizing carbide catalysts involving high-temperature annealing processes are also lack of effectiveness in controlling the size down to $\sim 3 \mathrm{~nm}$.[26-33]All these issues thus limit their practical industrial applications.

In this work, we prepared WC catalysts with the currently-reported smallest size of $1.9 \pm$ $0.9 \mathrm{~nm}$ with kilogram-scale production of $1.2 \sim 2.4 \mathrm{~kg} /$ day using a modified arc-discharging method in a flowing methane atmosphere.[34]The product is constituted of a high density of single $\mathrm{W}$ atoms, sub-nanometre and nanometre WC clusters, all of which are completely encapsulated in graphitic layers, thus providing exceptionally catalytic stability and activity in electrochemical reactions.

\section{Methods}

\subsection{Sample synthesis}


The sample was prepared by a modified arc-discharge method.[34]A high-purity tungsten rod forms the anode, attached to a water-cooled copper stage for rapid sample cooling, whereas the upper carbon rod serves as the cathode. The chamber is first evacuated to reach $10^{-2} \mathrm{~Pa}$ and, subsequently, methane $\left(\mathrm{CH}_{4}\right)$ is introduced as a gaseous carbon source to reach $0.1 \times 10^{5} \mathrm{~Pa}$. Evaporating the tungsten rod by arc-discharge at $\sim 30 \mathrm{~V}$ and $\sim 90 \mathrm{~A}$ results in a simultaneous decomposition of methane. The current and voltage are controlled by adjusting the distance between the tungsten rod and the carbon rod for continuous stable operation. During this evaporation process, we have noticed that the carbon rod has negligible loss compared with the carbon decomposed from the methane. The arc-evaporated tungsten and decomposed carbon form black smoke and simultaneously deposit on the cool walls of the chamber. The asfabricated product is collected after about $\sim 6 h$.

\subsection{Characterization}

The microstructures of $\mathrm{W}$-in-C and WC-in-C complexes were analyzed using a Hitachi S4700 transmission/scanning electron microscope operated at a voltage of $30 \mathrm{kV}$. STEM imaging and EELS were performed using an aberration-corrected STEM (Nion-UltraSTEM100) operating at a low accelerating voltage of $60 \mathrm{kV}$. The achievable spatial resolution using this fifth-order aberration corrected microscope at $60 \mathrm{kV}$ is $\sim 1.2 \AA$. ADF images were obtained using 58-200 mrad detector half-angle. EEL spectra were collected using a Gatan Enfina spectrometer with a collection half-angle of $48 \mathrm{mrad}$. The energy resolution of the EELS spectrum measured from the zero-loss full width at half-maximum was $0.3 \mathrm{eV}$. A Siemens D-500 X-ray diffractometer with $\mathrm{CuK \alpha}(\lambda=0.154 \mathrm{~nm})$ radiation at a voltage of $30 \mathrm{kV}$ and a current of $30 \mathrm{~mA}$ was used to study the phase structure of the products at a scan step of $0.2^{\circ}$.

\subsection{Theoretical simulation.}


The calculations have been performed using the Vienna ab initio simulation pack.[35] The projected augmented wave method and the Perdew-Wang generalized gradient approximation were used to describe electron-ion interactions. A thermal broadening of $0.1 \mathrm{eV}$ was applied to improve the convergence of the self-consistent procedure and non-spin polarization was employed for all calculations, except the $\mathrm{O}_{2}$ adsorption simulation with plane-wave cut-off energy of 50 Ry. For bulk WC, a 20×20×20 Monkhorst-Pack grid was used for the integration of the elemental unit cell over the Brillouin zones. The WC clusters with different sizes were located in the $25 \AA \times 25 \AA \times 25 \AA$ simulation box, and the stable configurations were obtained through full relaxation according to the Hellmann-Feynman forces.

\section{Results and discussion}

Herein we report an arc-discharge approach to synthesize the WC clusters with a mean diameter of $1.92 \pm 0.95 \mathrm{~nm}$, which is the smallest WC size in currently reported literatures. The ultra-small WC clusters are encapsulated completely in graphitic layers, thus presenting highlyperformance structural stability. Moreover, by an atomic-scale microscopy characterization, we observed a high density of single $\mathrm{W}$ atoms and abundant sub-nanometre crystalline and amorphous WC clusters in the product. These microstructural features endow WC@C composite compelling advantages as a highly-efficient catalyst, and meanwhile provide direct evidence on the correlation between the macroscopic electrochemical behaviors and the atomic-scale origin of the catalytic sites. In particular, our approach can reach a productivity of $50-100 \mathrm{~g}$ per hour, and thus has significant potentials in the development of WC catalysts for industrial needs.

The microstructures of WC@C composite were characterized by using a fifth-order aberration-corrected scanning transmission electron microscope (STEM, Nion-UltraSTEM100), coupled with an electron energy-loss spectrometer (EELS, Gatan Enfina spectrometer). The low 
accelerating voltage of $60 \mathrm{kV}$ avoids the electron beam induced damage to the ultra-small WC clusters and crystallization of some amorphous WC clusters and single W atoms, thus providing the reliable microstructural information.[36]Fig. 1A shows a representative bright-field (BF) image of WC@C composite, in which one cannot observe any heterogeneous phase due to the ultra-small size of WC clusters. However, these WC clusters can be clearly distinguished in the corresponding annular dark-field (ADF) image, shown in Fig. 1B, because the mass-thickness contrast differences, i.e., higher-density and/or heavier elements generate brighter contrast.[37, 38]With ADF imaging, the mean WC cluster diameter was estimated to be $1.92 \pm 0.9 \mathrm{~nm}$ (Fig. 1C) by a statistic analysis of more than $500 \mathrm{WC}$ clusters (except for dispersive single $\mathrm{W}$ atoms). ADF image shown in Fig. 1D presents the zoomed-in microstructures of one representative tungsten carbide nanoparticle, evidencing that the quantitative ratio of ADF intensity between the tungsten carbide particle and the graphite matrix is $\sim 2.62$ and the particle diameter of $\sim 2$ nm.

Fig. 2A and $\mathbf{B}$ shows the powder X-ray diffraction pattern and Raman spectroscopy of WC@C powders, verifying the components of $\mathrm{W}_{2} \mathrm{C}, \mathrm{WC}$ and graphite components. The total fraction of the W component determined by energy-dispersive X-ray (EDX) spectroscopy was measured to be 19.2 wt.\%. It should be noted that the fitted Raman spectroscopy shown in Fig. 2B presents four typical peaks (I, II, III and IV) at $1224.4 \mathrm{~cm}^{-1}, 1324.8 \mathrm{~cm}^{-1}, 1452.7 \mathrm{~cm}^{-1}$, and $1583.4 \mathrm{~cm}^{-1}$. The ones at $1324.8 \mathrm{~cm}^{-1}$ and $1583.4 \mathrm{~cm}^{-1}$ were thought to be from D band and $\mathrm{G}$ band ascribed to graphitic phases, while the ones at $1224.4 \mathrm{~cm}^{-1}$ and $1452.7 \mathrm{~cm}^{-1}$ can be attributed to the coexistence of $\mathrm{sp}^{2}$ and $\mathrm{sp}^{3}$ bonds, resulting from the high density of defects associated with serious lattice disorder at atomic scale.[39, 40]It evidently implies that the 
combined operation of arc-discharge evaporation and rapid-cooling non-equilibrium processes results in the diversity of product.

Fig. 3A and $\mathbf{B}$ show BF and ADF images of a region consisting of several WC clusters and a high density of single-atom $\mathrm{W}$ dispersion, respectively. The carbon matrix is composed of amorphous phase and severely distorted graphitic layers, which result in pronounced microstructure defects such as ripple, wrinkle and porosity. In the ORR catalytic process, it has been unambiguously proven that $\mathrm{O}_{2}$ molecules are more prone to penetrate and react at defective sites.[38, 41-47]Therefore, although the WC clusters and single-atom W-C complex are caged, we reasonably convince that such a highly-defective feature of graphitic layers also provide and even enhance the synthetic catalytic role.[48]Furthermore, the magnified regions and the Fast Fourier Transform(FFT) patterns in Fig. 3A, marked as A1, A2, A3 and A4, provide more detailed insights into the WC clusters, revealing atomic-scale structures, such as lattice distortion(A2), amorphous phase(A3) and even a high density of dispersed single-atom W(A4). It is well known that, the redistribution of $d$-band electrons of the metal atoms of carbides, ascribed to lattice expansions arising from the interstitial carbon atoms, is the origin of the Ptlike catalytic characteristics.[11-13]The observed microstructures unambiguously indicate that the lattice expansion of $\mathrm{W}$ atoms in the WC@C composite might be further enhanced due to these severe defects, particularly for the dispersed single-atom W-C complex. The WC@C synthesized herein provides multi-combined advantages as a synthetic catalyst.

In order to confirm the electrochemical catalytic properties of WC@C composite, the ORR properties were evaluated systematically in $\mathrm{N}_{2}$ - and $\mathrm{O}_{2}$-saturated $0.1 \mathrm{M} \mathrm{KOH}$ electrolytes by using an $\mathrm{Ag} / \mathrm{AgCl}$ three-electrode electrochemical cell. The WC@C composite was deposited on a rotated-disk glassy carbon $(\mathrm{GC})$ electrode $(5.6 \mathrm{~mm}$ in diameter) and the mass density was 
controlled at $60 \mu \mathrm{gcm}^{-2}$. Fig. 4A shows the cyclic voltammetry (CV) curves of the catalysts recorded in both electrolytes at room temperature and at a scan rate of $100 \mathrm{mVs}^{-1}$. It can be seen that, a well-defined cathodic $\mathrm{O}_{2}$ reduction current peak appears at $-0.1 \mathrm{~V} \sim-0.4$ in the $\mathrm{O}_{2}$-saturated $0.1 \mathrm{M} \mathrm{KOH}$ electrolyte, but it is absent in $\mathrm{N}_{2}$-saturated $0.1 \mathrm{M} \mathrm{KOH}$ electrolyte, proving the ORR catalytic activity of WC@C composite.

To uncover the electrochemical kinetic feature of WC@C composite during the ORR electrochemical process, polarization curves were carried out at a scan rate of $5 \mathrm{mVs}^{-1}$ and at different rotation rates ranging from 200 to $1400 \mathrm{rpm}$, as shown in Fig. 4B. The mass-transferlimited current density was evidently enhanced as increasing the rotation rate, associated with an onset potential at -0.23 V. Fig. 4 C shows the calculated Koutecky-Levich $(K-L)$ plots $\left(J^{-1}\right.$ vs. $\omega^{-}$ 1/2) based on the Koutecky-Levich $(\boldsymbol{K}-\boldsymbol{L})$ equations [Eq. (1-3)], in which the expected linearity and parallelism at all the potential simply first-order reaction kinetics. According to the $K-L$ plots, the limited diffusion currents $\left(\boldsymbol{J}_{\boldsymbol{K}}\right)$ and the electron transfer number (n) can be obtained:

$\frac{1}{J}=\frac{1}{J_{L}}+\frac{1}{J_{K}}=\frac{1}{B w^{1 / 2}}+\frac{1}{J_{K}}$

$B=0.62 n F C_{o}\left(D_{o}\right)^{2 / 3} v^{-1 / 6}$

$J_{K}=n F k C_{o}$

Where $\boldsymbol{J}$ is the measured current density, $\boldsymbol{J}_{\boldsymbol{K}}$ and $\boldsymbol{J}_{\boldsymbol{L}}$ are the kinetic- and diffusion-limiting current densities, $\boldsymbol{\omega}$ is the angular velocity of rotated-disk electrode, $\boldsymbol{n}$ is the overall number of electrons transferred in oxygen reduction, $\boldsymbol{F}$ is the Faraday constant $\left(\boldsymbol{F}=96485 \mathrm{C} \cdot \mathrm{mol}^{-1}\right), \boldsymbol{C}_{\boldsymbol{0}}$ is the bulk concentration of $\mathrm{O}_{2},\left(\boldsymbol{C}_{\boldsymbol{0}}=1.2 \times 10^{-3} \mathrm{~mol} \cdot \mathrm{cm}^{-3}\right), \boldsymbol{v}$ is the kinematic viscosity of the electrolyte $\left(v=0.01 \mathrm{~cm}^{2} \cdot \mathrm{s}^{-1}\right), \boldsymbol{D}_{0}$ is the diffusion coefficient of $\mathrm{O}_{2}$ in $0.1 \mathrm{M} \mathrm{KOH}\left(1.9 \times 10^{-5} \mathrm{~cm}^{2} \cdot \mathrm{s}^{-1}\right)$. The $\mathrm{n}$ and $J_{k}$ were calculated to be $3.8 \sim 4.2$ and $6.2 \sim 23.3 \mathrm{mAcm}^{-2}$ at the potential range of $-0.4 \mathrm{~V} \sim-0.8$ 
$\mathrm{V}$, respectively, as shown in Fig. 4D. It is proven the four-electron transfer process of oxygen reduction for WC@C catalyst.

The durability of the WC@C catalyst was evaluated by the normalized chronoamperometric curve recorded at $-0.40 \mathrm{~V}$ and a rotation rate of $1400 \mathrm{rpm}$ in an $\mathrm{O}_{2}$-saturated $0.1 \mathrm{M} \mathrm{KOH}$ electrolyte. As shown in Fig. 5A, the residual current density after 30000s measurement can effectively remain at $\sim 80 \%$, presenting more superior durability than that of graphene, mesoporous carbon and commercially Pt-C.[41-44]Furthermore, the anti-interfering experiments were carried out in the chronoamperometric measurement in the $\mathrm{O}_{2}$-saturated $0.1 \mathrm{M}$ $\mathrm{KOH}$ electrolyte, as shown in the inset of Fig. 5A. One cannot observe noticeable current change by the additions of $10 \mathrm{ml}$ methanol or ethanol in a $500 \mathrm{ml}$ electrolyte cell. Such high selectivity of the WC@C catalyst towards the ORR can effectively avoid crossover effects in polymer electrolyte fuel cells. In particular, the current density $\left(6.2 \sim 23.3 \mathrm{mAcm}^{-2}\right.$ at $\left.-0.4 \sim-0.8 \mathrm{~V}\right)$ and stability of $J_{k}(\mathbf{F i g} .5 \mathrm{~B}$ and $\mathbf{C})$ are comparable to that of other currently reported catalysts, such as commercially Pt-C $\left(5.1 \mathrm{mAcm}^{-2}\right)$,[41]graphene $\left(3.3 \sim 5.3 \mathrm{mAcm}^{-2}\right.$ for S-doped and 2.5 7.8 $\mathrm{mAcm}^{-2}$ for N-doped), [41] and born/nitrogen-doped CNTs $\left(5.4 \sim 9.5 \mathrm{mAcm}^{-2}\right)$.[43, 44]

As we known, the origin of the catalytic characteristics of carbides of groups IV through VI can be ascribed to the increased density of states (DOS) near the Fermi surface.[12] Here we calculated the electronic properties of WC clusters with different sizes and single W atoms and the iso-surfaces of the charge densities in a small energy window near the Fermi surface are shown in Fig.6A. It suggest that the d-electrons located both at external corners and edges of low-coordinated $\mathrm{W}$ atoms in the carbide particles, as well as the electrons on the single $\mathrm{W}$ atoms incorporated into the graphitic layers, result in an increased DOS near the Fermi surface. Fig.6B and $\mathbf{C}$ show the DOS of d-electrons of $\mathrm{W}$ and present much higher values for ultra-small clusters 
and single W atoms compared with bulk WC.

To reveal the catalytic ability of these single $\mathrm{W}$ atoms in ORR, a simplified model consisting of an $\mathrm{O}_{2}$ molecule adsorbed on the surface of the single $\mathrm{W}$ atom-doped graphite layer was characterized using spin-polarized first-principles calculations. We considered all the possible configurations of a single $\mathrm{W}$ atom at a single vacancy, bi vacancy, tri vacancy or quad vacancy of carbon atomic layer. In all the configurations that we proposed, the configuration shown in Fig.6D is the most probable site for $\mathrm{O}_{2}$ catalytic reduction. Accordingly, we studied the catalytic activity of an $\mathrm{O}_{2}$ molecule absorbed on this most probable configuration of a single W atom-carbon layer. The free $\mathrm{O}_{2}$ molecule was added close to the $\mathrm{W}$-carbon layer without any artificial assumption, such as the adsorption sites. By optimizing both the structure and energy at $300 \mathrm{~K}$, the $\mathrm{O}_{2}$ molecule seeks its most favourable absorption site and configuration, and bonds with the $\mathrm{W}$ atom after only $\sim 100 \mathrm{ps}(20,000$ steps $)$ simulation. The bond length of the $\mathrm{O}_{2}$ molecule was calculated to be $1.22 \AA$ and the atomic configuration of $\mathrm{O}_{2}$ adsorbed on the single W site was calculated, including spin polarization. The diatomic adsorption effectively weakens the $\mathrm{O}-\mathrm{O}$ bonding. The $\mathrm{O}-\mathrm{O}$ bond length and stretching frequency are computed to be $0.151 \mathrm{~nm}$ and $519 \mathrm{~cm}^{-1}$, significantly weaker than that of $\mathrm{O}_{2}$ at a perfect $\mathrm{Pt}[48]$ surface $(0.143 \mathrm{~nm}$ and 690 $\left.\mathrm{cm}^{-1}\right),[48]$ which thus facilitates the $\mathrm{O}-\mathrm{O}$ dissociation. From the iso-energy surfaces of electron densities differences shown in Fig.6D, an obvious charge transfer from the $\mathrm{W}$ atom to the two $\mathrm{O}$ atoms can be observed, associated with a filling of $\pi^{*}$ anti bonding states. This is the origin of the relatively low $\mathrm{O}-\mathrm{O}$ dissociation energy barrier. These atomic-scale insights confirm that the low-coordinated and single $\mathrm{W}$ atoms are the most active catalyst sites.

The chemical and structural stability of catalysts would be unavoidably reduced as decreasing the catalyst size, in particular down to sub-nanometer scale, which has seriously 
limited the optimization of catalytic activity. In the present study, ultrasmall WC catalysts are completely encapsulated within graphitic layers, and thus the agglomeration and coarsening of catalysts can be effectively suppressed during reaction processes. Moreover, high-defective graphitic layers, associated with a high density of sub-nanometre porous channels, significantly improve the penetration and exchange for $\mathrm{O}_{2}$ molecules, ions and electrolyte, as well as the overall conductivity, as illustrated in Fig. 7. These combined advantages therefore make WC@C catalysts simultaneously possess high-performance catalytic activity and stability.

\section{Conclusions}

In conclusion, the encapsulation architecture of WC@C catalysts not only prohibits the chemical/thermal coarsening of WC clusters, but the highly defective graphitic layers can also improve the overall conductivity and penetration capacities. These combined advantages of WC@C composite thus result in a competitive potential as a substitute of noble metal-based catalysts, and open up a new approach for developing non-noble metal-based catalysts. However, more theoretical and experimental works are also needed to fully understand the roles of individual $\mathrm{WC}, \mathrm{W}_{2} \mathrm{C}$ and graphitic phases, as well asto further optimize the catalytic performances of WC@C composite by modifying the arc-discharge approach in coming works.

\section{Acknowledgments}

JJG and HXZ gratefully acknowledge the National Natural Science Foundation of China (51501124) and Basic Research Project in Shanxi Province (2015021071, 2015011037). PFG gratefully acknowledge the support of the national 1000-plan for young scholars. XFZ acknowledges the support of National Natural Science Foundation of China (51471045), the national 1000-plan for young scholars, and the start-up funding supported from the Northeastern 
University of China. XFZ and GWQ thank Changjiang Scholars and Innovative Research Team in University (No. IRT0713). This research used facilities provided by Oak Ridge National Laboratory's Center for Nanophase Materials Sciences (CNMS), which is sponsored by the Scientific User Facilities Division, Office of Science, Basic Energy Sciences, U.S. Department of Energy.

\section{Notes and references}

[1] B.C. Steele, A. Heinzel, Nature 414 (2001) 345-352.

[2] R. Borup, J. Meyers, B. Pivovar, Y.S. Kim, R. Mukundan, N. Garland, D. Myers, M. Wilson, F. Garzon, D. Wood, Chemical reviews 107 (2007) 3904-3951.

[3] C. Burda, X. Chen, R. Narayanan, M.A. El-Sayed, Chemical reviews 105 (2005) 1025-1102.

[4] A.S. Arico, P. Bruce, B. Scrosati, J.-M. Tarascon, W. Van Schalkwijk, Nature materials 4 (2005) 366-377.

[5] L. Lin, Q. Zhu, A.-W. Xu, Journal of the American Chemical Society 136 (2014) 1102711033.

[6] B. Lim, M. Jiang, P.H. Camargo, E.C. Cho, J. Tao, X. Lu, Y. Zhu, Y. Xia, science 324 (2009) 1302-1305.

[7] H.A. Gasteiger, S.S. Kocha, B. Sompalli, F.T. Wagner, Applied Catalysis B: Environmental 56 (2005) 9-35.

[8]L. Xiao, L. Zhuang, Y. Liu, J. Lu, Journal of the American Chemical Society 131 (2008) 602608.

[9] Y. Bing, H. Liu, L. Zhang, D. Ghosh, J. Zhang, Chemical Society Reviews 39 (2010) 21842202. 
[10] D. Wang, Y. Li, Advanced Materials 23 (2011) 1044-1060.

[11] R. Levy, M. Boudart, science 181 (1973) 547-549.

[12] L. Bennett, J. Cuthill, A. McAlister, N. Erickson, R. Watson, Science 184 (1974) 563-565.

[13] J. Houston, G. Laramore, R.L. Park, Science 185 (1974) 258-260.

[14] H.H. Hwu, J.G. Chen, K. Kourtakis, J.G. Lavin, The Journal of Physical Chemistry B 105 (2001) 10037-10044.

[15] M. Neylon, S. Choi, H. Kwon, K. Curry, L. Thompson, Applied Catalysis A: General 183 (1999) 253-263.

[16] L. Delannoy, J.-M. Giraudon, P. Granger, L. Leclercq, G. Leclercq, Catalysis today 59 (2000) 231-240.

[17] I.J. Hsu, Y.C. Kimmel, Y. Dai, S. Chen, J.G. Chen, Journal of Power Sources 199 (2012) 46-52.

[18] Y. Liu, W.E. Mustain, ACS Catalysis 1 (2011) 212-220.

[19] Y.-W. Lee, A.-R. Ko, S.-B. Han, H.-S. Kim, K.-W. Park, Physical Chemistry Chemical Physics 13 (2011) 5569-5572.

[20] K. Yamamoto, T. Imaoka, W.-J. Chun, O. Enoki, H. Katoh, M. Takenaga, A. Sonoi, Nature chemistry 1 (2009) 397-402.

[21] A. Ullrich, Y. Chai, D. Pistorius, Y.A. Elnakady, J.E. Herrmann, K.J. Weissmann, U. Kazmaier, R. Müller, Angewandte Chemie 121 (2009) 4486-4489.

[22] B. Qiao, A. Wang, X. Yang, L.F. Allard, Z. Jiang, Y. Cui, J. Liu, J. Li, T. Zhang, Nature chemistry 3 (2011) 634-641.

[23] Y. Zhai, D. Pierre, R. Si, W. Deng, P. Ferrin, A.U. Nilekar, G. Peng, J.A. Herron, D.C. Bell, H. Saltsburg, Science 329 (2010) 1633-1636. 
[24] H. Zhang, T. Watanabe, M. Okumura, M. Haruta, N. Toshima, Nature materials 11 (2012) 49-52.

[25] Z. Huang, X. Gu, Q. Cao, P. Hu, J. Hao, J. Li, X. Tang, Angewandte Chemie 124 (2012) 4274-4279.

[26] V. Teixeira da Silva, E. Ko, M. Schmal, S. Oyama, Chemistry of materials 7 (1995) 179184.

[27] W.-F. Chen, C.-H. Wang, K. Sasaki, N. Marinkovic, W. Xu, J. Muckerman, Y. Zhu, R. Adzic, Energy \& Environmental Science 6 (2013) 943-951.

[28] Y. Zhao, K. Kamiya, K. Hashimoto, S. Nakanishi, Angewandte Chemie International Edition 52 (2013) 13638-13641.

[29] W.-F. Chen, J.T. Muckerman, E. Fujita, Chemical Communications 49 (2013) 8896-8909.

[30] S.T. Hunt, T. Nimmanwudipong, Y. Román - Leshkov, Angewandte Chemie International Edition 53 (2014) 5131-5136.

[31] R.W. Gosselink, D.R. Stellwagen, J.H. Bitter, Angewandte Chemie International Edition125 (2013) 5193-5196.

[32] Y. Liu, T.G. Kelly, J.G. Chen, W.E. Mustain, ACS Catalysis 3 (2013) 1184-1194.

[33] Z. Yan, M. Cai, P.K. Shen, Scientific reports 3 (2013) 1646.

[34] X. Zhang, J. Guo, P. Guan, C. Liu, H. Huang, F. Xue, X. Dong, S.J. Pennycook, M.F. Chisholm, Nature communications 4 (2013) 1924.

[35] G. Kresse, J. Furthmüller, Computational Materials Science 6 (1996) 15-50.

[36] O.L. Krivanek, M.F. Chisholm, V. Nicolosi, T.J. Pennycook, G.J. Corbin, N. Dellby, M.F. Murfitt, Z.S. Szilagyi, M.P. Oxley, S.T. Pantelides, Nature 464 (2010) 571-574.

[37] P. Nellist, S. Pennycook, Science 274 (1996) 413-415. 
[38] K. Gong, F. Du, Z. Xia, M. Durstock, L. Dai, science 323 (2009) 760-764.

[39] S. Choudhury, M. Agrawal, P. Formanek, D. Jehnichen, D. Fischer, B. Krause, V. Albrecht, M. Stamm, L. Ionov, ACS nano, 9 (2015) 6147-6157.

[40] A.C. Ferrari, J. Robertson, Philosophical Transactions of the Royal Society of London A: Mathematical, Physical and Engineering Sciences 362 (2004) 2477-2512.

[41] S. Yang, L. Zhi, K. Tang, X. Feng, J. Maier, K. Müllen, Advanced Functional Materials 22 (2012) 3634-3640.

[42] Z. Chen, D. Higgins, Z. Chen, Carbon 48 (2010) 3057-3065.

[43] S. Wang, E. Iyyamperumal, A. Roy, Y. Xue, D. Yu, L. Dai, Angewandte Chemie50 (2011) 11756-11760.

[44] R. Liu, D. Wu, X. Feng, K. Müllen, Angewandte Chemie49 (2010) 2565-2569.

[45] M. Lefèvre, E. Proietti, F. Jaouen, J.-P. Dodelet, science 324 (2009) 71-74.

[46] T. Cui, R. Lv, F. Kang, Q. Hu, J. Gu, K. Wang, D. Wu, Nanoscale research letters 5 (2010) 941-948.

[47] Y. Li, W. Zhou, H. Wang, L. Xie, Y. Liang, F. Wei, J.-C. Idrobo, S.J. Pennycook, H. Dai, Nature nanotechnology 7 (2012) 394-400.

[48] A. Eichler, J. Hafner, Physical review letters 79 (1997) 4481-4484. 

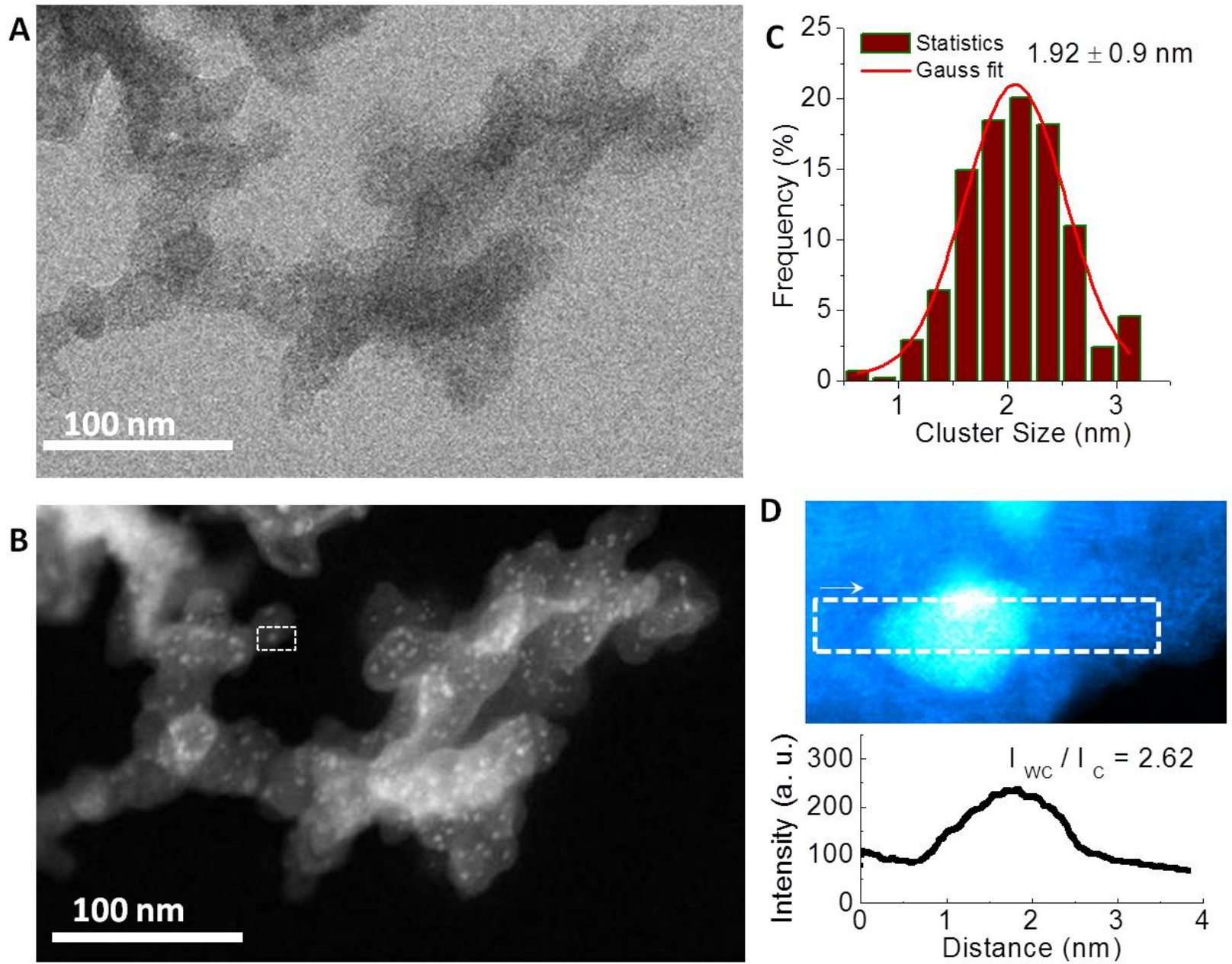

Fig.1 Microstructural characterizations of WC@C catalysts. A, Bright-field STEM image of WC@C complex composite. B, ADF image of the same region as Fig.1A, exhibiting ultrasmall tungsten carbide particles (bright points) that are unobvious in the $\mathrm{BF}$ image. $\mathbf{C}$, the size distribution of WC clusters. D, One representative tungsten carbide cluster presents the evident contrast difference compared with the graphitic layer matrix, in which the ADF intensity was quantitatively measured as marked by a white square. 

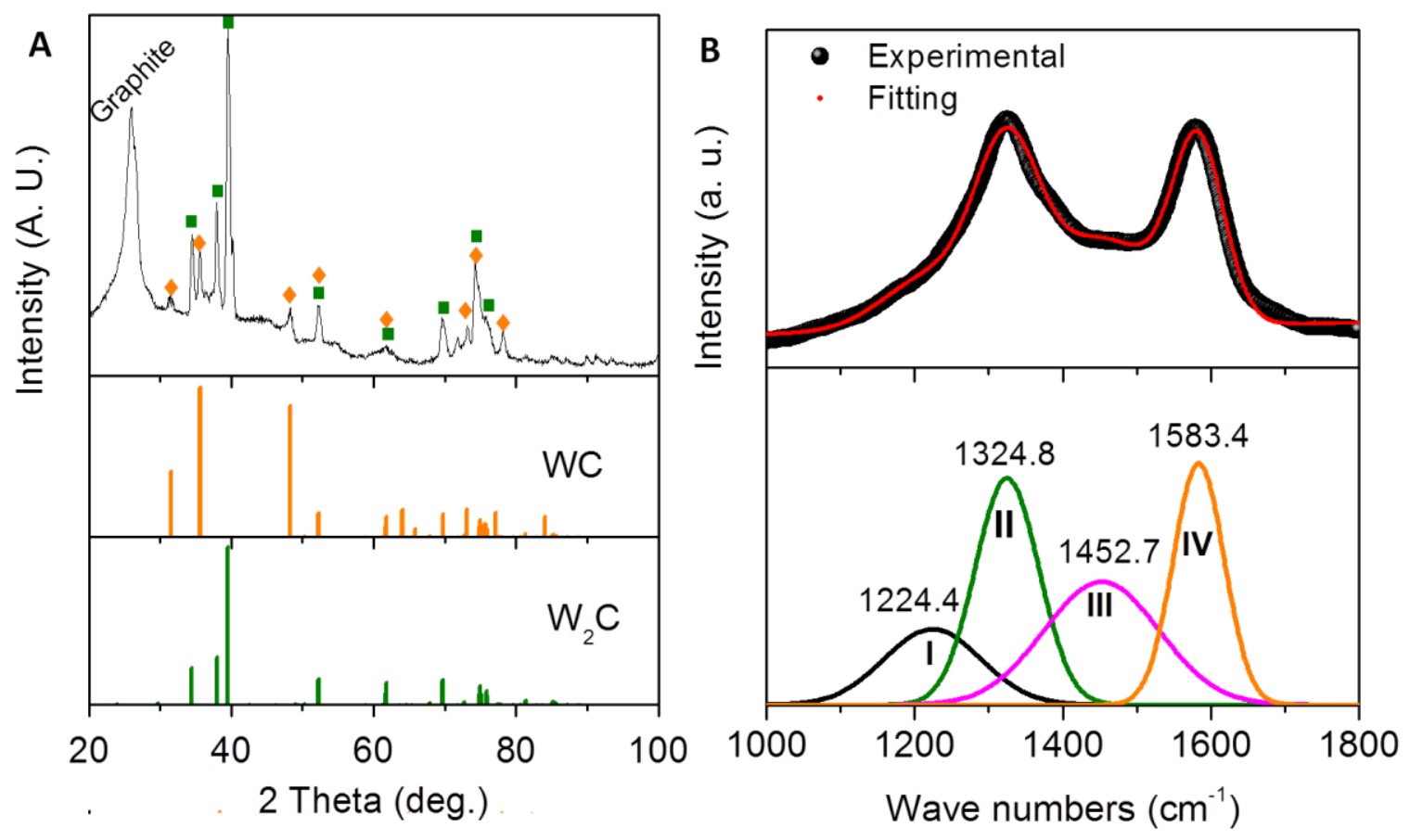

Fig. 2 Microstructural characterizations of WC@C catalysts. A, X-ray diffraction pattern of WC@C powders, showing that the product is composed of $\mathrm{WC}_{2}, \mathrm{WC}$, and graphite phases. $\mathbf{B}$, Raman spectroscopy of WC@C powders. Noted that the fitted peaks (I, II, III and IV) at 1224.4 $\mathrm{cm}^{-1}, \quad 1324.8 \mathrm{~cm}^{-1}, 1452.7 \mathrm{~cm}^{-1}$, and $1583.4 \mathrm{~cm}^{-1}$ can be attributed to the signatures of nanocrystalline diamond, D band, and fullerenes, and G band, respectively. 

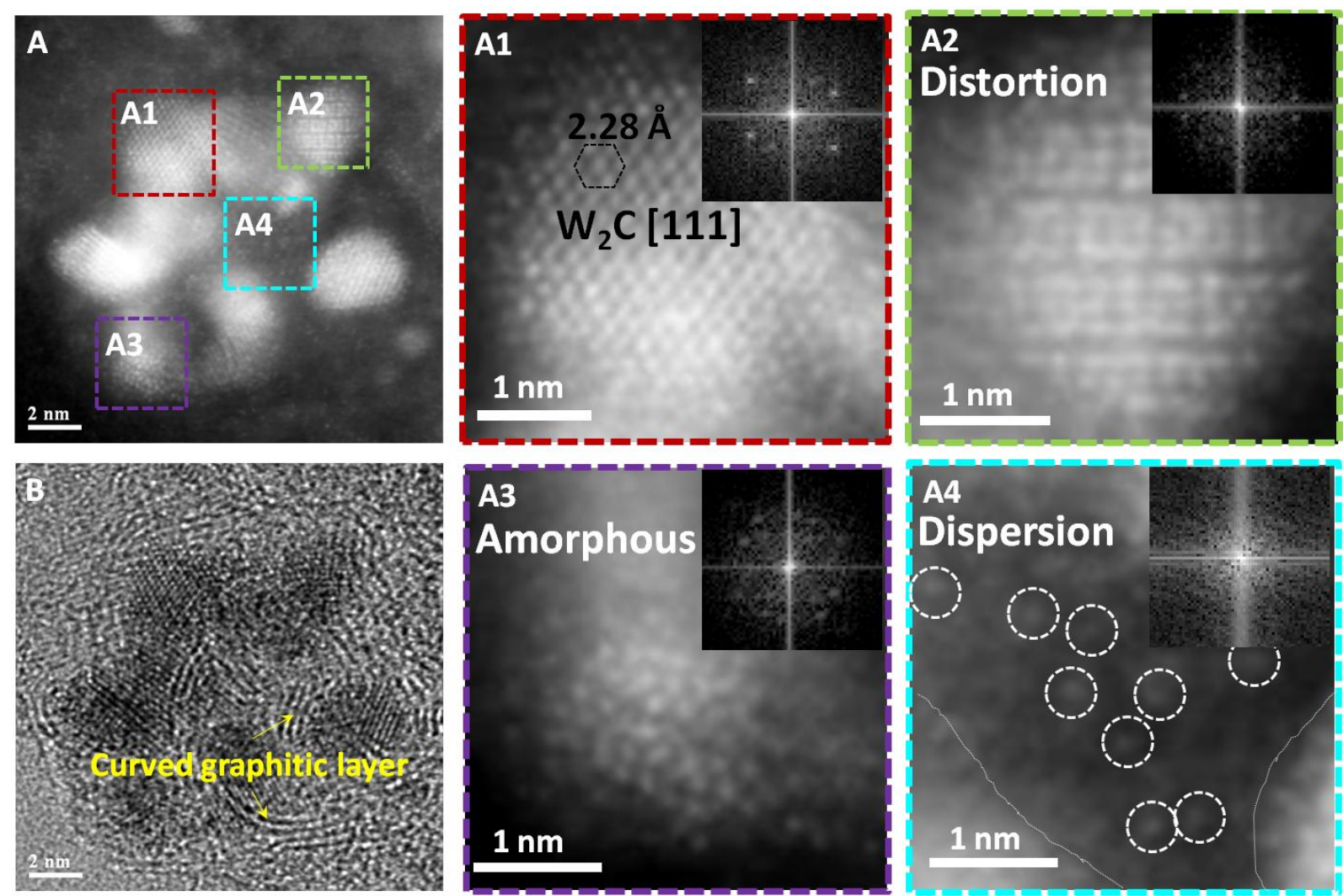

Fig. 3 Microstructures of WC@C catalysts. A and B, ADF image and the corresponding STEM image of WC@C complex consisting of several WC clusters, in which four representative regions are identified. Enlarged A1, A2, A3 and A4 images and the FFT patterns of the regions reveal atomic structures of WC clusters encapsulated in the graphitic layers, such as lattice distortion (A2), amorphous state (A3) and even some dispersed single tungsten atoms (A4) those are marked by white circles. 

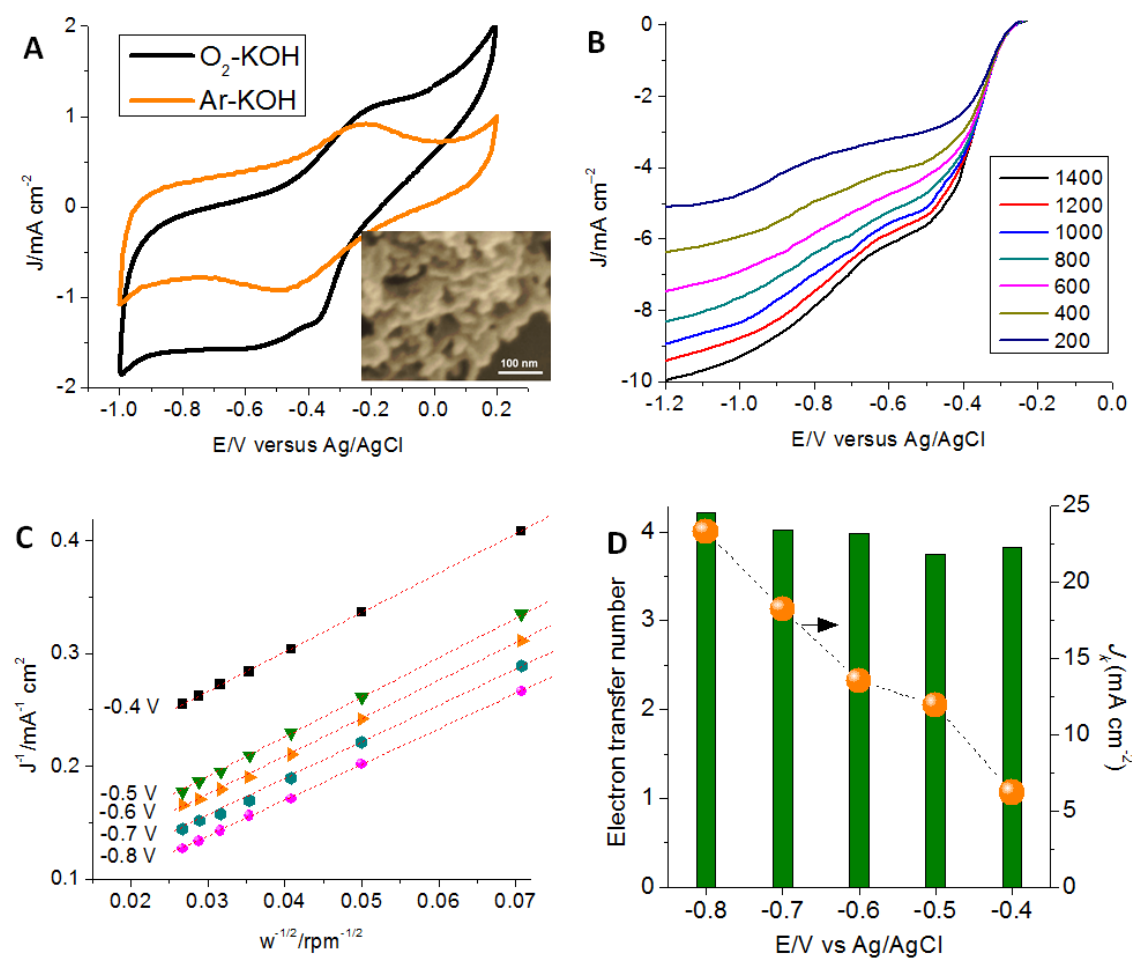

Fig.4 Evaluation of ORR activity performances of WC@C catalysts. A, Cyclic voltammograms $(\mathrm{CV})$ curves of ORR on WC@C complex in a $\mathrm{N}_{2}$-saturated 0.1M KOH solution, an $\mathrm{O}_{2}$-saturated $0.1 \mathrm{M} \mathrm{KOH}$ solution at a scan rate of $100 \mathrm{mVs}$. The inset in Fig.4A is the Scanning electron microscopy (SEM) image of deposited WC@C catalyst electrode. B, Linear sweep voltammetry curves recorded for WC@C complex in an $\mathrm{O}_{2}$-saturated $0.1 \mathrm{M} \mathrm{KOH}$ solution at a scan rate of $5 \mathrm{mVs}^{-1}$ and different rotation rates. C, Koutecky-Levich plot of $J^{-1}$ versus $w^{-1 / 2}$ at different electrode potentials. D, The dependence of electron transfer number $(n)$ on potential and the Kinetic-limiting current density $\left(J_{k}\right)$ at $-0.4 \mathrm{~V}$. 

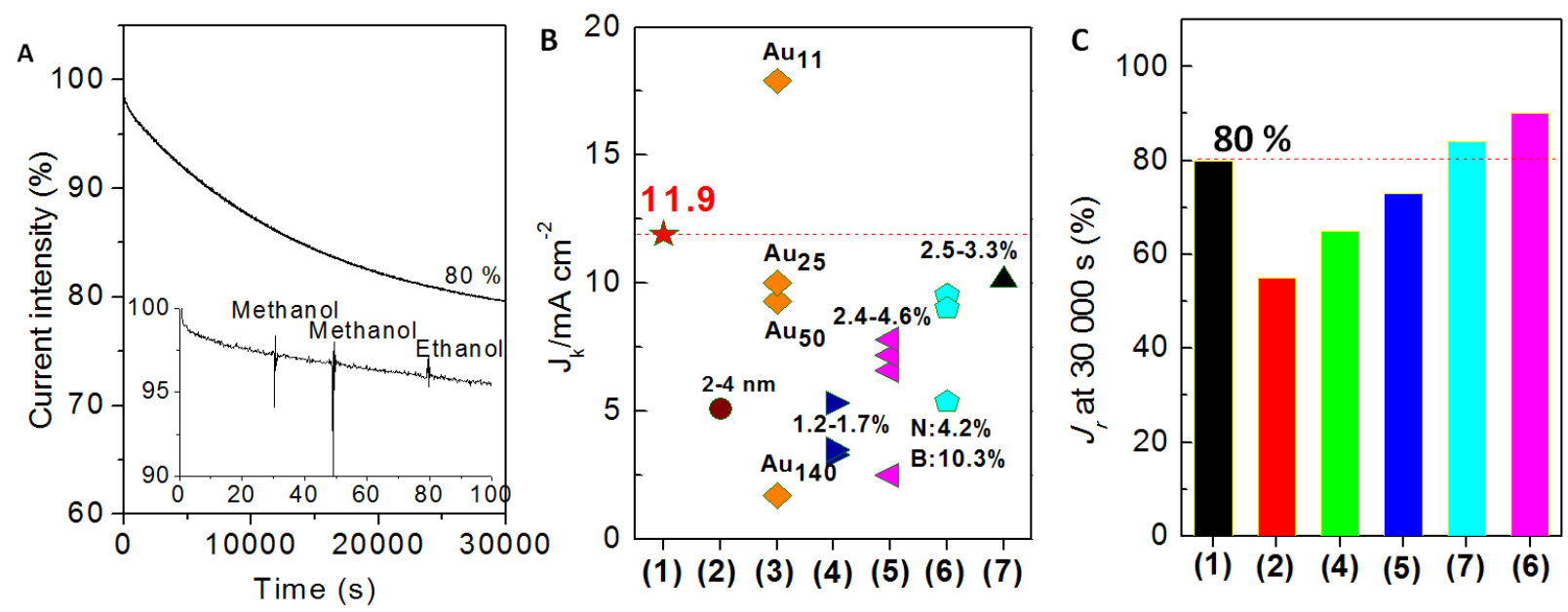

Fig.5 Evaluation of ORR stability performances of WC@C catalysts. A, Chronoamperometric response curve of WC@C complex recorded at $-0.5 \mathrm{~V}$ in an $\mathrm{O}_{2}$-saturated $0.1 \mathrm{M} \mathrm{KOH}$ at a rotation rate of $1400 \mathrm{rpm}$. The inset in $\mathbf{A}$, Chronoamperometric response curve was further performed under the addition of methanol and ethanol, evidencing the electrocatalytic selectivity of WC@C complex. B and C, Kinetic-limiting current stability and density $\left(J_{k}\right)$ at $-0.5 \mathrm{~V}$ in comparison with that of the reported studies. The reference numbers (1), (2), (3), (4), (5), (6),(7) and (8) correspond to the present WC@C complex, commercial Pt nanoparticles[40], Au clusters [21], sulfur-doped graphene[40], nitrogen-doped grapheme[40], nitrogen-doped carbon nanotubes[42], nitrogen/boron-doped carbon nanotubes[42], and nitrogen-doped mesoporous carbon [43], respectively. Noted that the $J_{k}$ value of BN-CNT is calculated at $-0.3 \mathrm{~V}$ and the others are at $-0.5 \mathrm{~V}$. 


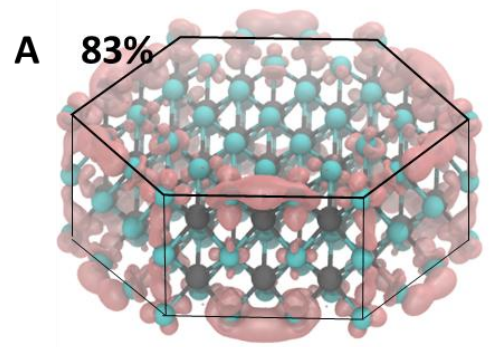

Large Size: 1nm

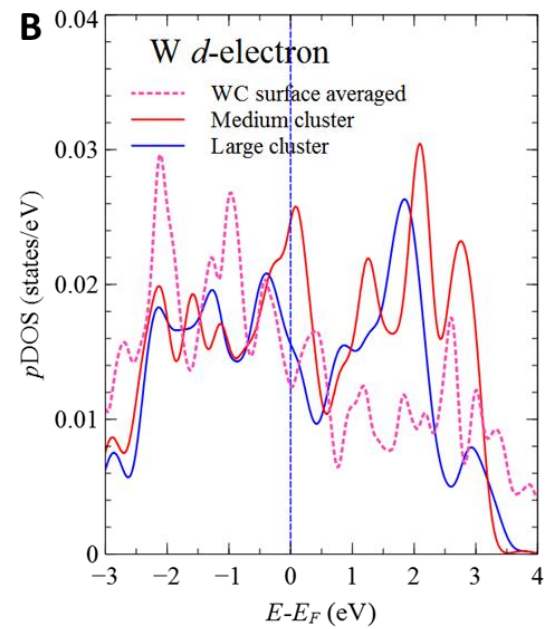

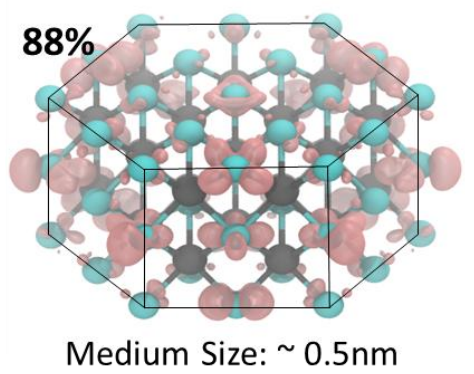

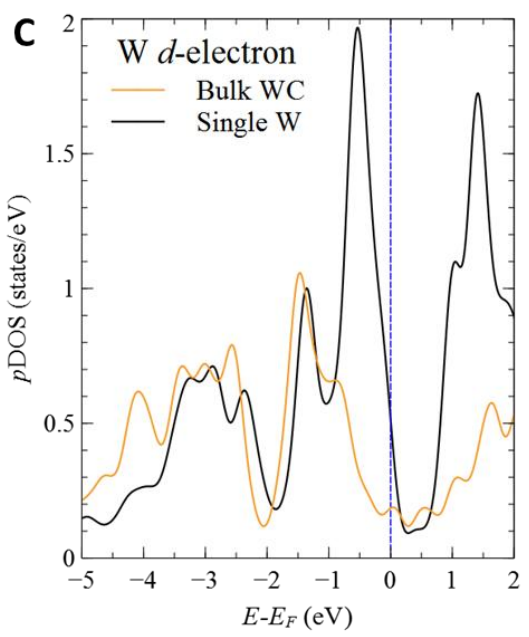

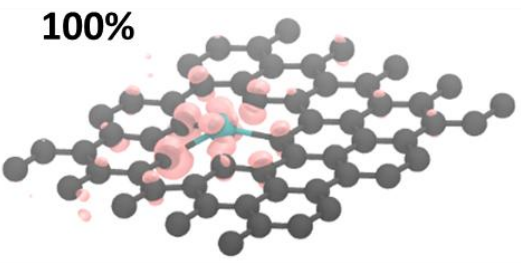

Single W Atom
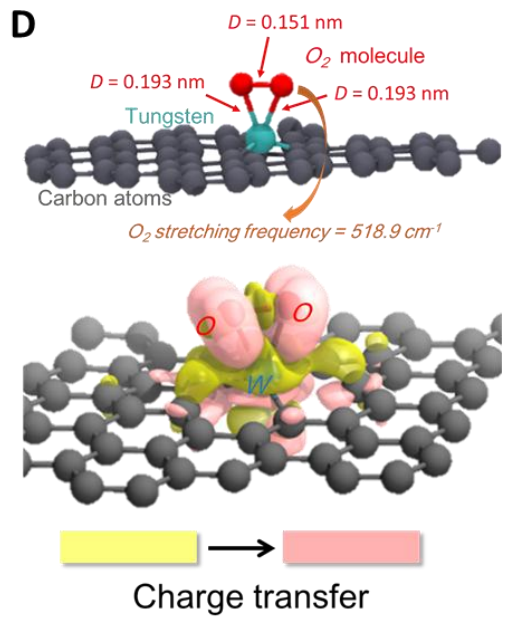

Fig.6 Theoretical simulation of the catalytic origins. A, The electronic properties of WC clusters with different sizes and single $\mathrm{W}$ atoms and the iso-surfaces of the charge densities in a small energy window near the Fermi surface. $\mathbf{B}$ and $\mathbf{C}$ show the DOS of d-electrons of W and present much higher values for ultra-small clusters and single W atoms compared with bulk WC. D, Isosurface of the difference in electron density before and after $\mathrm{O}_{2}$ adsorption. 


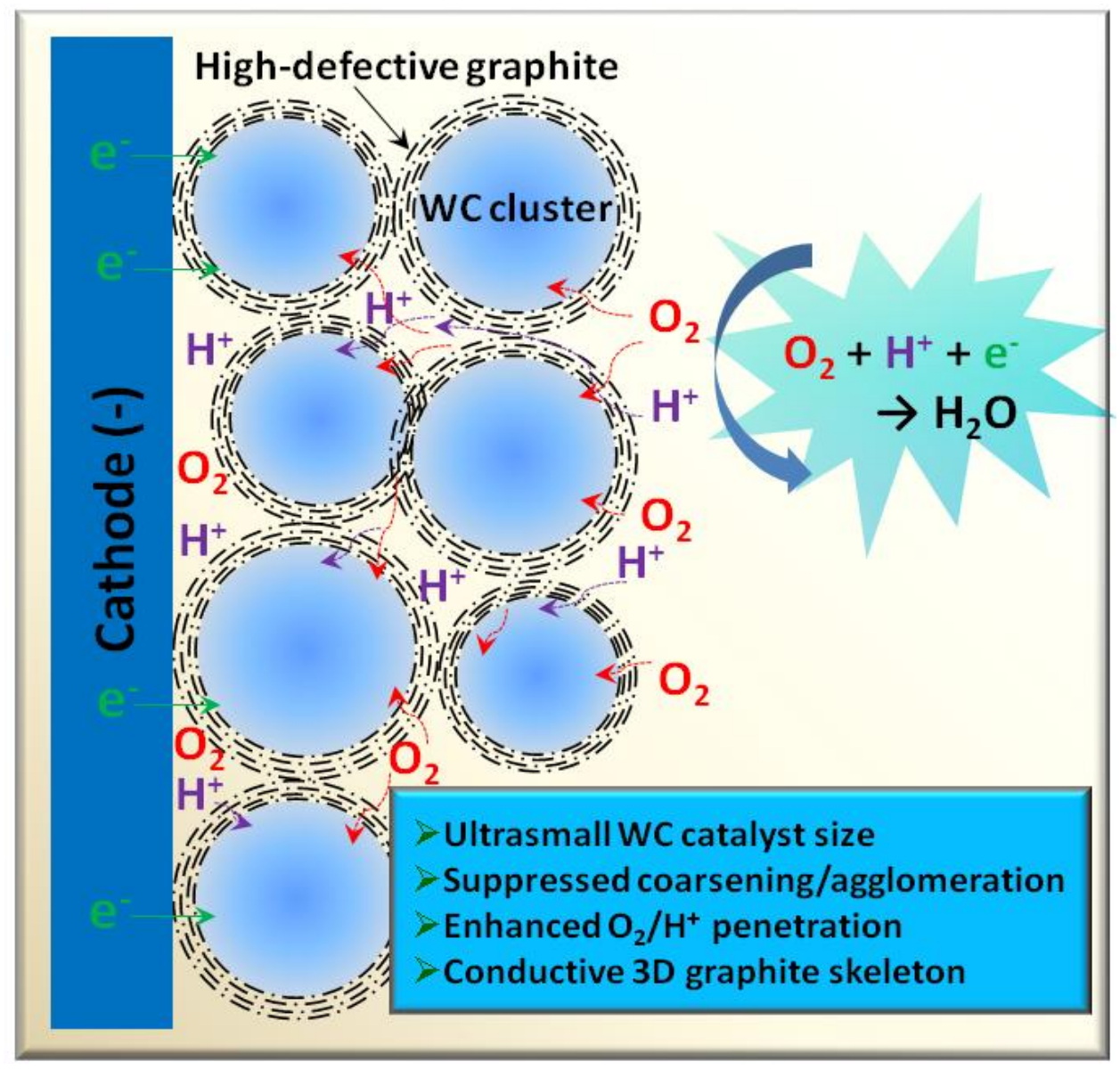

Fig.7 Illustration for WC@C catalysts as the catalytic cathode. WC catalysts are completely encapsulated within high-defective graphitic layers, which improve the catalytic stability by suppressing the crystalline growth and agglomeration of WC clusters, and meanwhile enhance the penetration of oxygen molecules, ions and electrolyte during the reaction processes, as well as the formation of three dimensional conductive skeletons. 


\section{Graphical abstract}

Ultrasmall Tungsten Carbide Catalysts Stabilized in Graphitic Layers

for High-performance Oxygen Reduction Reaction

Junjie Guo, Zhe Mao, Xiaoli Yan, Rui Su, Pengfei Guan, Haixia Zhang, Bingshe Xu, Xuefeng Zhang, Gaowu Qin, Stephen J. Pennycook
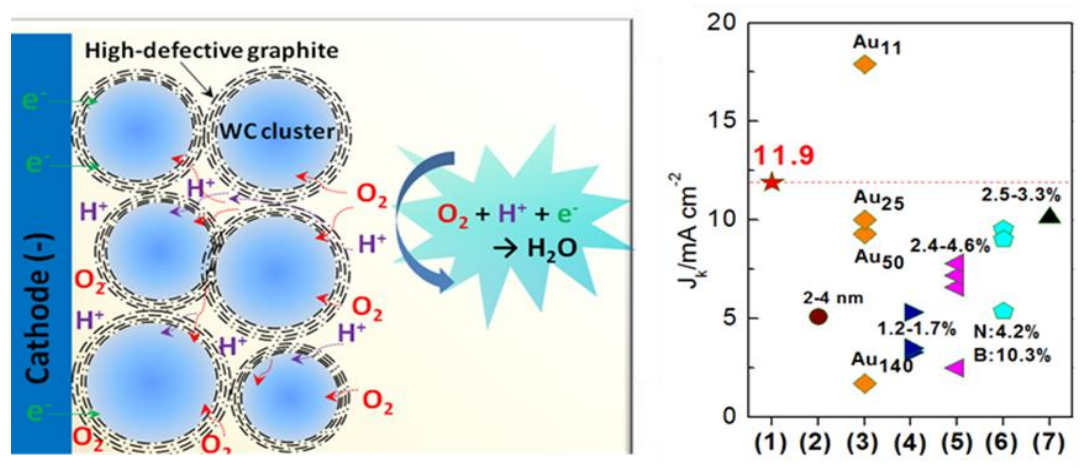

We synthesized WC catalysts with a mean diameter of $1.9 \pm 0.9 \mathrm{~nm}$, comprising a high density of single $\mathrm{W}$ atoms, sub-nanometer and nanometer WC clusters, which are completely encapsulated in high-defective graphitic layers $(\mathrm{WC} @ \mathrm{C})$. Such high-defective graphitic layers not only improve the overall conductivity for accelerating the penetration and exchange of ions and electrons during the electrocatalytic process of oxygen reduction, but also suppress the chemical/thermal coarsening of WC catalysts, thus providing excellent electrocatalytic performances in four-electron transfer process for oxygen reduction reaction. 